ÉGYPTE

monde arabe

\section{Égypte/Monde arabe}

14 | 1993

Dits et écrits, mémoires et rites

\title{
Dits et écrits, mémoire et rites. Introduction
}

Iman Farag

\section{OpenEdition}

\section{Journals}

Édition électronique

URL : https://journals.openedition.org/ema/550

DOI : 10.4000/ema.550

ISSN : 2090-7273

\section{Éditeur}

CEDEJ - Centre d'études et de documentation économiques juridiques et sociales

\section{Édition imprimée}

Date de publication : 30 juin 1993

Pagination : 7-14

ISSN : 1110-5097

\section{Référence électronique}

Iman Farag, «Dits et écrits, mémoire et rites. Introduction », Égypte/Monde arabe [En ligne], 14 | 1993,

mis en ligne le 08 juillet 2008, consulté le 07 juillet 2022. URL : http://journals.openedition.org/ema/ 550 ; DOI : https://doi.org/10.4000/ema.550

Ce document a été généré automatiquement le 7 juillet 2022

Tous droits réservés 


\title{
Dits et écrits, mémoire et rites. Introduction
}

\author{
Iman Farag
}

\author{
«L'écriture est la possibilité du jeu de l'intellect \\ sur la langue. » \\ J. Bazin, A. Bensa, Introduction à Jack Goody, La \\ raison graphique, Minuit, 1985
}

1 Au départ, la perspective était celle d'une livraison dont les articles seraient réunis par une démarche commune - celle de l'anthropologie. A l'arrivée, on constate que l'unité de l'ensemble a opéré là où on l'attendait le moins. C'est surtout de par les objets dont ils traitent que les divers articles qui composent ce numéro se trouvent ordonnés autour de quelques axes. A leur tour, ils recomposent un champ disciplinaire. Du coup, les questions suscitées portent autant sur les objets proprement dits que sur la logique qui préside à leur découpage et à leur sélection et sur l'ensemble qui en constitue l'aboutissement.

2 Mythes, figures et espaces. A deux reprises la ville de Louxor impose son visage paradoxal; une fois à travers son saint patron Abu-l-Hajjaj (R. Chih), une autre fois à travers ses conteurs populaires (R. Schautz). Mais si l'on y regarde de plus près, est-ce si paradoxal ? Que le lieu par excellence de la rencontre avec l'altérité - touristique soit en quelque sorte un musée/conservatoire des arts et traditions populaires, cela semble aller de soi. Valorisation du patrimoine, folklorisation, mais au-delà de l'action des acteurs urbains louxoriotes, résidents ou de passage, il nous faut aussi questionner le regard des chercheurs.

3 La figure d'Abu-l-Hajjaj ressort d'autant mieux, confrontée à celle d'Ibn al-Fârid (P-J. Luizard). Deux mawlid pour deux figures de saints, mais qui s'inscrivent dans des durées et des espaces ô combien différents. Plus fondamentalement, c'est sur leurs places respectives dans les mémoires qu'on peut réfléchir; l'un, vivant grâce au culte rendu par une ville qui perpétue sa mémoire, l'autre, vacillant sauf dans les mémoires de ses fidèles, qui maintiennent le culte du saint mort contre vents et marées de l'oubli. Que la beauté du mort (M. de Certeau) l'emporte - sur la laideur du vivant? - rien de plus 
humain. Cependant, une fois tracées les figures de saints, les images de mawlid, il faudrait tenter le parallèle qui en fait deux objets possibles et complémentaires et, là aussi, interroger la démarche des chercheurs.

4 Si les saints assurent la médiation avec le divin, deux articles se penchent en contrepoint sur des figures de la médiation profane ; conteurs populaires et écrivains publics (M. Doss) assurent des rôles sociaux qui vont au-delà de la simple performance du premier et de la compétence du second. Cependant, dans un cas comme dans l'autre - et c'est cela qui permet le rapprochement et le rend d'autant plus intéressant - le monopole sur le savoir détenu est tout relatif. L'auditoire du conteur sait déjà .ce qu'il va écouter, et recourir à l'écrivain public ne veut pas dire forcément être analphabète. Autant dire que de l'esthétique à l'utilitaire, tout est question de style. Et de légitimité.

5 Passons de la prise de parole comme situation au contenu du message. Des conteurs de Louxor à la geste populaire et ce qu'elle véhicule (Ali Fahmi). Avec tous les risques que cela implique, l'accent se déplace du comment au pourquoi et il n'est plus question de performance mais de la geste populaire comme réservoir de la mémoire collective. Notion controversée, difficilement opératoire certes mais qui, dans la galerie des portraits, permet d'opérer tris et classements ; plus que des héros, Baybars et 'Ali al-Zaybaq sont ce qui permet de reconstituer l'anti-histoire; non pas intemporelle mais carrément inversée, qui récompense le juste, châtie le tyran et préfigure le futur rêvé. La geste populaire est représentée ici comme une stratégie de résistance. La démarche qui consiste à confronter ou juxtaposer geste populaire et chroniques anciennes peut-elle frayer une voie vers l'histoire des mentalités (une histoire qui ne peut être que sociale) ? La question reste posée.

6 Il sera souvent question, dans certains articles (conteurs populaires, geste populaire, fête populaire...), d'égyptianité et de personnalité nationale (un avatar du populaire ?). On est certes en droit de poser la question de la persistance de concepts non opératoires, jugés obsolètes, de mauvaise réputation ou franchement "infamants", comme celui de personnalité nationale. Mais il faudrait auparavant considérer leurs fonctions dans les luttes socio-politiques à l'intérieur d'une société donnée et bien plus, dans la manière dont celle-ci se situe dans un temps, un espace et un système-monde (encore une obsolescence !). L'intérêt d'une telle notion - s'il en est - est peut-être d'inciter au retour sur soi; sur les modes d'énonciation de l'identité de soi du chercheur, ou d'une non-identité auto-proclamée.

7 Les deux articles qui complètent cet ensemble portent sur les pratiques enfantines dans le Khan al-Khalîlî (A. Madœuf) et le rituel de la divination dans le zâr (T. Battain). Il n'ont aucun point commun si ce n'est cette incertitude à propos de l'accès à la parole des acteurs et ce que l'on en fait. Parole d'enfant ou parole de possédé, autant dire qu'on a affaire à des quasi méta-langages. Le fossé ne peut être comblé que par l'exploitation de toutes les ressources (et énergies) de l'observation. Celles-ci ne sont pas illimitées. Et la question posée porte sur la place que se choisira l'observateur, si choix il y a : quel qu'il soit, quelle qu'elle soit, il y aura toujours une part de perte.

Mythe, figure, espace

8 Parole dite, parole écrite... Parole donnée, pourrait-on ajouter : Madiha Doss s'intéresse à la fonction de l'écrivain public aujourd'hui en Égypte, au cœur même du Caire. Son interrogation va bien au-delà du contenu des messages échangés, des registres de langue mobilisés et des collusions que peuvent occasionner les passages de l'oral à l'écrit. Au centre de tout cela se trouvent des «conditions d'énonciation» qui 
distribuent et figent les rôles et déterminent les règles du jeu; s'agissant de l'écrivain public, c'est bien un pacte social tacite qui le rend possible. D'entrée de jeu, l'auteur refuse la facilité et ce n'est pas vers une problématique de l'illettrisme que M. Doss s'oriente, ni vers celle d'une opposition entre l'écrit et l'oral, mais vers une problématique de la médiation, à double palier si l'on peut dire, puisque le rapport à l'écrit semble fortement conditionner le rapport à l'écrivain public qui, à son tour, du simple fait de sa compétence scripturaire réelle ou supposée, se trouve investi d'une panoplie de rôles sociaux.

Allant à l'encontre des idées reçues, l'auteur souligne que tous les clients de l'écrivain public ne sont pas, loin s'en faut, des illettrés. Que les requêtes d'écriture relatives à la vie privée ne touchent qu'un nombre infime de clients qui préfèrent s'adresser à l'intermédiaire privé de leur choix. Pour un service gratuit, ou pour ne pas avoir à dévoiler sa vie privée ? Peut-être bien les deux. On sait par ailleurs que depuis quelques années, grâce au boom du musaggel (enregistreur), une cassette remplace avantageusement - la Poste a prévu un service spécial - la lettre reçue ou envoyée au frère, ou au mari travaillant au Koweït, en Libye ou à Paris. Les clients sont surtout tels que les décrit Madiha Doss - ni plus ni moins que d'honorables citoyens qui, troublés par la perspective d'une quelconque démarche administrative, si anodine soitelle, en perdent leur arabe. Remplir un formulaire, écrire une attestation qu'il faudra par la suite faire estampiller, ou plus encore adresser une plainte à l'administration, est une opération à laquelle on se prépare. Même ceux qui maîtrisent l'écriture reconnaissent à l'écrivain public une capacité dont ils se pensent dépourvus et lui accordent la confiance et la légitimité qu'ils se refusent.

Du coup, le médiateur est aussi censeur; il code, arrondit les angles, met les formes pour faire bref et efficace, tout en restant fidèle au sens. Intermédiaire entre «Monsieur le Gouvernement » et le peuple, traducteur de l'oral vers l'écrit, on peut imaginer le degré de confiance dont il est investi. On peut se demander également si, pour des raisons d'éthique, l'écrivain public peut refuser une commande. En amont, il écoute et compatit - choses que les fonctionnaires de l'administration d'à côté n'ont ni le temps ni l'envie de faire; en aval, il sait, quand il le faut, garder un secret. Les frontières entre sphères publique et privée s'en trouvent certes redéfinies, mais chacun y trouve son compte. Le Graphique a ses raisons que la Raison ne comprend pas.

11 Est-ce une autre raison qui commande le rôle du conteur populaire étudié par Rosine Schautz ? Les éléments du dispositif restent tout à fait comparables à ceux décrits par Madiha Doss. Car au cœur de la "raison graphique » et de la "raison orale », il y a un même élément central: l'attente. Sans attente, il n'est point de conteurs; loin de constituer un tout homogène, on peut identifier parmi les conteurs des grades, selon qu'ils se montrent poètes, chantent, parlent ou racontent; bref, en fonction non seulement de la maîtrise d'un art codé mais de leur capacité à innover; d'où l'importance de questionner cette part de créativité - d'individuation? - qui passe à travers ce qu'on serait tenté d'envisager comme une simple réitération. Mais le conte est un peu comme les vagues d'une mer, jamais les mêmes : l'auditoire fait partie de la performance et le corps du conteur, plus qu'un simple support du message, s'investit et se laisse envoûter par sa parole. Eléments qu'on ne retrouvera plus une fois le conte figé sur cassette. Celle-ci ne réussit pas non plus à recréer ce " plus » que seule apporte la présence du conteur; convivialité et récréation de l'espace des représentations 
communes, constitutives de la mémoire collective égyptienne, si l'on admet de donner un rôle à cette catégorie.

En complément à ces deux articles figure dans ce numéro (cf. rubrique "Lettrés arabes ", dans la partie Traduire] un essai de traduction d'Iman Farag. Il s'agit de fragments de Qantara alladhi kafar, roman doublement unique de Mustafa Mucharrafa, parce qu'il n'en a pas écrit d'autres et parce que, de mémoire de roman égyptien contemporain, il est le seul à s'être exprimé, d'un bout à l'autre, en dialectal.

Avatars de Louxor ou de son saint patron: des deux, qui a fabriqué l'autre? C'est la question qui s'impose à la lecture des notations de Rachida Chih sur Abu-l-Hajjaj. Que ce soit dans le mythe constitutif de l'image du saint ou dans le mawlid qui le célèbre, on peut lire la spécificité d'une histoire - une durée - régionale, marquée tant par sa géographie que par l'accumulation de strates historiques visibles à l'œil nu. D'AbulHajjaj, le savant - chroniqueurs et biographes - et le populaire - tradition orale donnent des images différentes. Soufi thaumaturge et extatique ou soufi muhaddith et réformateur des mœurs, il y a peu de sens à s'interroger sur la «vraie " vie d'AbulHaijaj, mais tout amène à réfléchir sur l'interface entre les deux mythes. Interface qui à son tour donnerait à penser ce dédoublement que l'on peut constater au cœur même du mawlid entre la maqsûra - espace de dévotion - et l'espace ludique, celui des balançoires, des confiseries, des courses et... des conteurs. Ils sont peut-être cette forme liminaire qui permet de dépasser la simple juxtaposition entre la fête - la jouissance et l'ascèse.

De quoi nous ramener à Ibn al-Fârid, « sultan des amoureux ». Pierre-Jean Luizard a lui aussi tenté de reconstituer les survivances parallèles d'Ibn al-Fârid et la manière dont elles se retrouvent et s'expriment à travers son mawlid. Si Abu-l-Hajjaj reste vivant dans les mémoires grâce à sa mosquée (dans sa ville) - pour que la baraka se transmette il faut un lieu, comme le note R. Chih - à quoi Ibn al-Fârid doit-il sa survie ? Et d'abord, est-ce de la survie du saint ou du poète qu'il s'agit? La polémique que son diwân continue de susciter est encore autre chose. Résistant à l'oubli ou à l'occultation (les deux ?), son mawlid est celui d'un saint mort - cela se passe dans la Cité des morts -, l'on y récite sa poésie et l'on s'y transperce gaiement les joues. Ibn al-Fârid semble davantage survivre dans les mémoires de ses fidèles par son diwân que par ses hauts faits. Et au sein du mawlid il semble aisé de faire la différence entre le public habituel de ce genre de manifestations, les gens du quartier - majoritairement des enfants - et ceux qui sont venus là pour réciter ou écouter sa poésie (en plus de deux photographes curieux et d'un chercheur français).

Cela n'a pas empêché le mawlid de tomber en désuétude. A cela s'ajoute une certaine ambiguïté du côté de l'establishment d'al-Azhar ou du Ministère des Waqfs. L'image du saint, dont on connaît très peu la vie mais dont on connaît la poésie - iconoclaste - en est-elle la cause ? Ou est-ce parce qu'a fait défaut la confrérie qui aurait permis d'entretenir son mawlid ? Chose pour laquelle, comme on le voit, la mémoire à elle seule ne suffit pas. La réponse est quelque part entre ces deux hypothèses. Il est sans doute très facile de dire - mais peut-on avancer autre chose ? - que la mémoire ne se survit pas à elle-même. Il lui faut un substrat social. Et culturel. La déchéance du saint mort vue à travers son mawlid et sa suivie vue à travers son diwân, éclairent plus qu'elle ne remettent en cause un processus. Cela s'appelle modernité.

16 Mais il ne s'agit pas ici de dresser la liste des incompatibilités. Pas plus pour Ibn al-Fârid que pour la divination dans le rituel du zâr. A lire l'article de Tiziana Battain, on est 
frappé par la prédominance de l'aspect technique: la magie aurait-elle perdu sa magie ? Le zâr, opération consistant à "calmer les esprits " et la divination, sorte de diagnostic préliminaire visant à les identifier... L'emploi de la métaphore médicale n'est pas fortuit, puisqu'on a recours aussi bien à la kud'iyya, au médecin qu'à l'homme de Dieu (cheikh ou prêtre). Qu'il s'agisse du diagnostic musical menant à la transe et préparant à la grande épreuve, ou de la prise de contact avec les esprits au travers des rêves, le tout - rituels, codes et imagerie - permettant l'interprétation constitue un corpus. Loin d'être figé, celui-ci comme tout savoir se renouvelle, certains éléments, tombant en désuétude, étant remplacés par d'autres. Telle qu'elle est vécue au travers de ces rituels en Égypte, la relation avec les esprits relève plus de la cohabitation - non exempte de conflits - que de l'exorcisme. A chacun son double.

Reste enfin à noter ici ce qui fait la particularité de la contribution d'Anna Madœuf ; pour une fois - qui n'est pas coutume - il s'agit bien d'observer les pratiques des enfants dans le Khan al-Khalîlî, enfants perçus comme acteurs à part entière alors que nos recherches tendent le plus souvent à les prendre comme cible privilégiée de l'action ou de la perception des adultes. Dire que les sociabilités sont constitutives de parcours, ou que les parcours sont jalonnés par des sociabilités virtuelles - les deux propos se complètent -, cela va de soi. Le Khan al-Khalîlî a cela de particulier qu'il ne s'agit pas d'une zone d'habitat; du coup, de par la nature même de cet espace - tant sa morphologie que les représentations que l'on s'en fait - sont exclues certaines des activités quotidiennes pratiquées dans l'espace d'un quartier. Au Khan, les enfants viennent soit pour travailler, soit pour répondre à l'attraction de ce qui constitue un centre-ville de substitution. On n'y Joue pas au foot, on ne fait que passer. Ce qui fait la spécificité d'une représentation et d'une pratique enfantines de cet espace est peutêtre cette impression de foule, de profusion et de gigantisme. Question de taille. Sans doute faut-il aller voir aussi dans ce qui est décrit comme une absence de frontières entre le ludique et l'utilitaire et ses répercussions sur l'appropriation d'un espace; comme le note A. Madœuf, activités, jeu, promenade, « errance » ne sont pas figés et ne s'excluent pas. De quoi donner à réfléchir - sur leurs propres pratiques - aux adultes. L'ensemble que constitue cette livraison d'Égypte/Monde arabe appelle quelques remarques. Nos auteurs ont tous fait appel à l'observation. Certains d'entre eux ont énoncé les règles $\mathrm{du}$ « jeu » dans lequel ils ont été pris, d'autres non.

Il est possible cependant d'émettre quelques hypothèses. Notamment quand, à son insu, l'observation se transforme en participation, avec tout ce qu'elle recèle de richesses insoupçonnées par l'outsider et... de pièges.

Il y aurait également à s'interroger sur la manière dont l'érudition vient à la rescousse de l'intuition (et de la construction). L'orientalisme de toujours - tel qu'en témoignent certains des articles - continue de faire bon ménage avec l'observation à chaud de l'ici et maintenant. Jusqu'où aller pour explorer les profondeurs de champ tout en évitant les anachronismes? Jusqu'où tirer le fil du temps long quand l'observation, elle, est - ou se veut - immédiate (mot qui veut dire aussi sans médiation) ? Et comment déconstruire sans la mutiler une construction savante quand cela devient la condition sine qua non pour construire son objet?

21 Resterait enfin à poser la question du retour sur soi que ce type de démarches rend d'autant plus nécessaire. C'est aussi la question des risques de la monographie, qui tendrait quelquefois à dégager son objet du faisceau de rapports dans lesquels il s'inscrit et des pièges du récit. Ces deux problèmes renvoient directement à celui de 
l'écriture ethnographique, c'est sa légitimité même qu'il faut à chaque fois fonder, sans sacrifier à des artifices scientistes, sans sacrifier (à) la subjectivité de l'écrivant. Il serait trop facile de réduire cette question de subjectivité à un simple jugement de valeur innocent, glissé au détour d'une phrase. C'est dans la construction même de ce qui fait l'objet de la recherche qu'elle se niche. Autrement dit, la question de méthode - qui est aussi une question épistémique - posée une fois de plus dans ce numéro, pourrait être formulée ainsi : comment garder sa part à l'intuition tout en respectant la démarche d'objectivation? 